Revista de Psicología Vol. 39 (2), 2021 (e-ISSN 2223-3733)

\title{
Eventos locales, efectos globales: emociones trascendentes e identificación con toda la humanidad
}

\author{
José J. Pizarro ${ }^{1}$, Laura Alfaro-Beracoechea ${ }^{2}$, Olaia Cusi ${ }^{3}$, Manuel L. Ibarra ${ }^{4}$, \\ Larraitz Zumeta ${ }^{5}$ y Nekane Basabe ${ }^{61 \mathrm{f}}$ \\ Universidad del País Vasco-España $a^{13,5,6}$, Universidad de Guadalajara-México ${ }^{2}$, \\ Universidad Autónoma del Estado de México ${ }^{4}$
}

Ante los desafíos del mundo globalizado, investigaciones recientes sugieren que la identificación con la humanidad tiene bases socio-afectivas y es impulsada con la celebración de rituales colectivos que promueven la benevolencia y universalismo. Este trabajo analiza la relación de una emoción de auto-trascendencia (Awe) vivida en encuentros colectivos locales

1 Licenciado de Psicología (Universidad de Talca) y doctor en Psicología Social (Universidad del País Vasco UPV/EHU). Miembro del grupo consolidado "Cultura, Cognición y Emoción”. Dirección postal: Avenida de Tolosa, 80, 20018, Donostia/San Sebastián, Guipúzcoa, Espańa. Contacto: jose.pizarro@ehu.eus. https://orcid.org/0000-0001-9883-8765

2 Doctora en Psicología con Orientación en Calidad de Vida y Salud, Profesora de Tiempo Completo en Universidad de Guadalajara, miembro del Sistema Nacional de Investigadores 2020 - 2022. Miembro de Cuerpo Académico Psicología Social, Bienestar e Intersubjetividad. Dirección postal: Departamento de Comunicación y Psicología, Av. Universidad 1115, Col. Lindavista, Ocotlán, Jalisco, México. Contacto: nadhielii.alfaro@académicos. udg.mx. https://orcid.org/0000-0002-9857-7339

3 Graduada en Psicología por la Universidad del País Vasco (UPV/EHU), estudiante de doctorado y miembro del grupo consolidado "Cultura, Cognición y Emoción”. Dirección postal: Avenida de Tolosa, 80, 20018, Donostia/San Sebastián, Guipúzcoa, España. Contacto: ocusi001@ikasle.ehu.eus. https://orcid.org/0000-0002-4518-1824

4 Licenciado en Psicología, maestro en C. en Salud en el Trabajo y Doctor en C. en Salud Colectiva (Universidad Autónoma Metropolitana-Xochimilco). Miembro del cuerpo académico consolidado "Biopsicología, Salud y Sociedad". Dirección postal: Av. Bordo de Xochiaca S/N col. Benito Juárez, C.P. 57000, Nezahualcóyotl, Estado de México. Contacto: mlibarrae@ uamex.mx. https://orcid.org/0000-0003-2492-186X

5 Doctora en Psicología e Investigadora en la Universidad del País Vasco. Dirección postal: Avenida de Tolosa, 80, 20018, Donostia/San Sebastián, Guipúzcoa, Espańa. Contacto: larraitznerea.zumeta@ehu.eus.

6 Profesora Catedrática de Psicología Social en la Universidad del País Vasco (UPV/EHU), España. Miembro del grupo consolidado de investigación en Psicología Social. Dirección postal: Avenida de Tolosa, 80, 20018, Donostia/San Sebastián, Guipúzcoa, Espańa. Contacto: nekane.basabe@ehu.eus. https://orcid.org/0000-0003-4753-4299 
con la identidad humana. Los resultados de ecuaciones estructurales a partir de un estudio transversal realizado en México y el País Vasco $(N=656)$ muestra que estas emociones incrementan la identificación con la humanidad, el bienestar (directamente) y también la conducta prosocial (de manera indirecta). Además, la intensidad de esta emoción también predice positivamente la percepción de sincronía emocional aun controlando por tendencias más estables de personalidad. En línea con la teorización de Durkheim, los procesos emocionales vividos en eventos colectivos fomentan el desarrollo de identidades compartidas y la promoción del bien común, a través de experiencias de auto-trascendencia, la cual puede resultar de gran importancia para la coordinación de esfuerzos comunes ante problemas sociales de gran envergadura.

Palabras clave: Identidad global, Emociones de Auto-Trascendencia, Valores de Trascendencia, Sincronía Emocional Percibida, Prosocialidad.

\section{Local Events, Global Effects: Transcendent Emotions and Identification with All Humanity}

Confronted with the challenges of the globalized world, recent research suggests that identification with humanity has socio-affective roots and is driven by the celebration of collective rituals that promote benevolence and universalism. This paper analyzes the relation of a self-transcendent emotion (i.e., Awe) lived in local collective meetings with human identity. We conducted a across-sectional study in Mexico and the Basque Country $(\mathrm{N}=656)$, and the results of structural equation modelling shows that this emotion increases the identification with humanity, well-being (directly) and prosocial behavior (indirectly). Further, the intensity of this emotion also predicted the perception of emotional synchrony while controlling for more stable and trait-alike variables. Following Durkheim, the emotional processes experienced in collective events encourage the development of shared identities and the promotion of the common welfare through experiences of self-transcendence, which can result of great importance in the common efforts to deal with current large-scale social issues.

Keywords: Global Identity, Self-Transcendent Emotions, Transcendent Values, Perceived Emotional Synchrony, Prosociality.

\section{Eventos locais, efeitos globais: emoçóes transcendentais e identificaçáo com toda a humanidade}

Diante dos desafios do mundo globalizado, pesquisas recentes sugerem que a identificação com a humanidade tem bases socioafetivas e é impulsionada pela celebração de rituais coletivos que promovem a benevolência e o universalismo. Este trabalho analisa a relação de uma emoção de autotranscendência (temor) vivenciada em encontros coletivos locais com a identidade humana. Os resultados das equaçóes estruturais de um estudo transversal realizado no México e no País Basco $(\mathrm{N}=656)$ mostram que essas emoçóes aumentam a identificação com a humanidade, o bem-estar (diretamente) e também o comportamento pró-social (indiretamente). Além disso, a intensidade dessa emoção também prediz positivamente a percepçáo de sincronia emocional, mesmo controlando tendências de personalidade mais estáveis. Em consonância com a teorização de Durkheim, os processos emocionais vividos em eventos coletivos promovem o desenvolvimento de identidades compartilhadas e a promoção do bem comum, por meio de experiências de autotranscendência, que podem 
Eventos locales, efectos globales: emociones trascendentes e identificación / Pizarro et al.

ser de grande importância para a coordenação de esforços comuns. problemas sociais em grande escala.

Palavras-chave: Identidade Global, Emoçóes de Autotranscendência, Valores de Transcendência, Sincronia Emocional Percebida, Prosocialidade.

\section{Événements locaux, effets mondiaux: émotions transcendantes et identification à toute lhumanité}

Face aux défis du monde globalisé, des recherches récentes suggèrent que l'identification à l'humanité a des bases socio-affectives et est animée par la célébration de rituels collectifs qui favorisent la bienveillance et l'universalisme. Ce travail analyse la relation d'une émotion de dépassement de soi (Awe) vécue lors de rencontres collectives locales avec l'identité humaine. Les résultats d'équations structurelles d'une étude transversale menée au Mexique et au Pays basque ( $\mathrm{N}=656)$ montrent que ces émotions augmentent l'identification à l'humanité, le bien-être (directement) et aussi les comportements prosociaux (indirectement). En outre, l'intensité de cette émotion prédit également positivement la perception de la synchronie émotionnelle, même en contrôlant des tendances de personnalité plus stables. Conformément à la théorisation de Durkheim, les processus émotionnels vécus dans les événements collectifs favorisent le développement d'identités partagées et la promotion du bien commun, à travers des expériences d'auto-transcendance, qui peuvent être d'une grande importance pour la coordination des efforts communs face à des problèmes sociaux à grande échelle.

Mots clés: identité globale, émotions d'auto-transcendance, valeurs de transcendance, synchronisation émotionnelle perçue, prosocialité. 
Tradicionalmente, el estudio de la construcción de las identidades que tienen las personas se ha hecho con relación a las diversas influencias que estas reciben. Así, es posible enumerar una gran cantidad de variables que tienen un impacto en el proceso de formación de las identidades de las personas, siendo estas influencias directas o indirectas, como antecedentes, o como resultado. Entre ellas destaca la importancia del mantenimiento de una fuente de gratificación asociada a la pertenencia a diversos grupos sociales, tal como es descrita a través de la perspectiva de la identidad social (Tajfel \& Turner, 1979; Turner, Hogg, Oakes, Reicher, \& Wetherell, 1987; para una revisión, ver Hornsey, 2008). Por otra parte, y tomando la perspectiva desde un análisis macro, también se encuentran el efecto de los patrones culturales en la elaboración del self social (Markus \& Kitayama, 1991, 2010), y, desde un análisis micro, los periodos del desarrollo asociados a su construcción (e.g., Zacarés, Iborra, Tomás, \& Serra, 2009).

El estudio de estas influencias y de cómo se organizan diversas identidades (e.g., de clase, deportivas, nacionales, étnicas, etc.) (e.g., Roccas $\&$ Brewer, 2002) son fundamentales para comprender la construcción de las identidades sociales y cómo estas impactan otros procesos psicológicos. De esta manera, se pueden realizar análisis más detallados sobre la manera en que -por ejemplo- se pueden fomentar identidades más inclusivas (Hornsey \& Hogg, 1999, 2002), o cómo nos adaptamos a nuevos contextos socioculturales en procesos migratorios (e.g., Urzúa, Basabe, Pizarro, \& Ferrer, 2017; ver Benet-Martínez \& Hong, 2014).

Sin embargo, vivimos en un contexto cambiante caracterizado por diversos factores que, hace tan solo 100 años no suponían una gran influencia (e.g., medios de comunicación masivos y transporte), por lo que estos modelos también deberían ser actualizados. De esta manera, la influencia de factores que antańo no se cuestionaban, pueden hoy provocar el fortalecimiento y/o activación de un nivel de categorización social que ha generado un creciente interés tanto en lo académico como 
en la opinión pública en general. Este nivel, que ya había sido descrito en la teoría de la categorización social (i.e., identidad humana) (Turner et al., 1987) puede representar un desafío para las culturas locales y tradicionales, tanto occidentales como orientales, creando un grado de "ambigüedad cultural" en el que el significado, las relaciones sociales y los valores se vuelven inciertos y variables (Arnett, 2002; Rosenmann, Reese, \& Cameron, 2016).

Por otra parte, y de gran importancia para las relaciones interpersonales en este nuevo paradigma, recientes investigaciones basados en modelos funcionales de las emociones (e.g., Fischer \& Manstead, 2008; Keltner \& Haidt, 1999; Niedenthal \& Brauer, 2012) muestra como estas son capaces de favorecer procesos de formación grupal pudiendo incluso estar relacionadas con las identidades superordinadas y más inclusivas (Stellar et al., 2017; Van Cappellen \& Rimé, 2014).

Es por estas razones que el presente artículo busca explicar las relaciones entre distintas experiencias emocionales (i.e., emociones de auto-trascendencia) con el desarrollo de identidades más inclusivas (i.e., supra-ordinadas), a la vez que promueve el estudio ético del cosmopolitismo con el fin de la promoción de un bienestar común (de Rivera, 2018; Liu \& Macdonald, 2016).

\section{Identidades Inclusivas y Emociones de Auto-Trascendencia}

\section{Identificación con toda la bumanidad}

Uno de los axiomas más ubicuos de la psicología social es la importancia que tiene la pertenencia a diversos grupos sociales. Estas identidades son fuentes de autoestima y permiten satisfacer diversas necesidades básicas, tales como la de pertenencia o la seguridad. Desde el enfoque de la identidad social, y específicamente de la categorización social, Turner y colaboradores (1987) describieron distintos niveles de auto-categorización en función de la inclusión de nuestros selfs: el personal, el social, y el humano. Sin embargo, no ha sido hasta más recientemente que este tema ha recibido mayor atención en estudios empíricos -muchas 
veces bajo diversos nombres. Por ejemplo, este nivel de identificación ha sido estudiado con el nombre de Identidad Social Global (Buchan et al., 2011), Identificación con Toda la Humanidad (McFarland, Webb, \& Brown, 2012), Ciudadanía Global (Reysen \& Katzarska-Miller, 2013), o Identidad Global (de Rivera \& Carson, 2015).

Diversos estudios empíricos muestran que, en general, la activación de este nivel de identificación social produce efectos sociales positivos. Casos como los estudios de Wohl y Branscombe (2005), o de Nickerson y Louis (2008) mostraron efectos positivos en las relaciones intergrupales, mientras que aquellos de Buchan y colaboradores (2011), por una parte, y McFarland y colaboradores (2012), por otra, revelaron una mayor preocupación por temas de alcance global como la ayuda humanitaria. Sin embargo, este nivel de identidad puede también estar a la base de efectos no deseados, como lo es una mayor justificación a delitos y actos inmorales cometidos por miembros del endogrupo (ver Greenaway \& Louis, 2010; Greenaway, Quinn, \& Louis, 2011). Por lo tanto, mayor importancia cobra la necesidad de mejorar la comprensión de las fuerzas que la pueden motivar y también, de los efectos que puede inducir.

En esta línea, se considera de gran relevancia la perspectiva de los estudios de de Rivera (de Rivera, 2018; de Rivera \& Carson, 2015), que se enfocan en la importancia de una identificación superordinada basada en lazos socioafectivos, y la posibilidad de generar rituales que permitan su celebración. En otras palabras, que la identificación con la humanidad posee una alta carga emocional, puede estar apoyada en valores que promueven la benevolencia, y que su celebración sería clave para su mantenimiento. Así, el fortalecimiento de este nivel de identidad requeriría de ciertas prácticas comunales (e.g., rituales y encuentros colectivos) que se pueden construir desde una perspectiva que promueva lo éticamente "correcto", a su vez que enaltece los elementos que une a las personas de distintos contextos (ver de Rivera, 2018).

\section{Awe como una emoción de auto-trascendencia}

Una línea teórico-empírica que es capaz de clarificar la relación entre la creación de identidades más inclusivas y diversas experiencias, 
motivaciones o fases del desarrollo, es el estudio de la auto-trascendencia del yo. Esta línea se puede analizar desde diferentes factores como etapas y/o fases de desarrollo (e.g., Maslow, 1964; Frankl, 1966) o como motivaciones personales (e.g., Schwartz, 1994) que, de manera última, orientan el self individual hacia "afuera". En otras palabras, hacia las necesidades de otros.

De esta manera, existirían diversas manifestaciones de experiencias auto-trascendentes (ver Yaden, Haidt, Hood, Vago, \& Newberg, 2017) y entre ellas, las Emociones de Auto-Trascendencia (EAs, de aquí en adelante), las cuales promueven un cambio desde la auto-absorción y las metas más relacionadas al propio self, hacia las del resto (Van Cappellen \& Rimé, 2014; ver también Fredrickson, 2013). Estas emociones se centran en dos atributos fundamentales: son producidas por estímulos que no están directamente orientados hacia el self individual, y porque promueven la unión con otras personas y grupos sociales (Haidt, 2003b; Stellar et al., 2017; Van Cappellen \& Rimé, 2014). Diversos ejemplos de estas emociones son el asombro maravillado (awe en inglés) (Keltner \& Haidt, 2003), la inspiración moral (elevation, en inglés) (Haidt, 2003a) y el Kama Muta (del sánscrito, sentirse conmovido por amor) (Fiske, Schubert, \& Seibt, 2016; ver también Fiske, Seibt, \& Schubert, 2017).

El asombro maravillado (de aquí en adelante, awe) es una emoción que se desencadena ante la percepción de algo grandioso, sublime o extremadamente poderoso (i.e., vastedad) (Keltner \& Haidt, 2003; Schurtz et al., 2012), pudiendo provocar así una necesidad de acomodar la información que se está procesando debido a que desafía el marco usual de la persona que la vive. Esta emoción suele ser reportada ante la percepción de estímulos -entre otros- como una maravilla natural, una experiencia de lo divino o la grandiosidad de un líder (Keltner \& Haidt, 2003; Shiota, Keltner, \& Mossman, 2007).

Como emoción colectiva, el awe involucra ciertos procesos cognitivos que llevan al individuo a ser parte de algo más grande que el yo, es decir, al disminuir el sentido del $Y o$, permite a los individuos orientarse hacia el colectivo y adherirse a entidades sociales más grandes. En este 
sentido, diversos estudios han demostrado que aquellos sujetos que experimentaron awe, observando, por ejemplo, paisajes y bellezas naturales (e.g., parques, montańas, etc.) informaron sentir una conexión más fuerte con otras personas (Bai et al., 2017), o incluso se sentían parte de entidades colectivas más amplias como su grupo, nación o especie humana (Shiota, Keltner, \& Mossman, 2007).

Del mismo modo, estudios recientes han documentado que, si bien nos conecta con entidades más grandes que uno mismo, la experiencia emocional de awe también nos orienta a conductas que promueven los intereses de los demás, y puede predecir un aumento de comportamientos de ayuda hacia extraños, donaciones de caridad y voluntariado (Guan, Chen, Chen, Liu, \& Zha, 2019; Piff, Dietze, Feinberg, Stancato, \& Keltner, 2015; Prade \& Saroglou, 2016; Rudd, Vohs, \& Aaker, 2012).

Finalmente, recientes avances en el estudio de esta emoción documentan los beneficios que supone sobre la salud y el bienestar. Así, se ha observado que las experiencias extraordinarias y cotidianas de awe, podrían ser un mecanismo para la mejora de la satisfacción momentánea en la vida (Anderson, Monroy, \& Keltner, 2018; Rudd, Vohs, $\&$ Aaker, 2012) y mayor bienestar diario (Gordon et al., 2017; Rudd et al., 2012). En conjunto, estas líneas de investigación independientes encuentran que el awe se relaciona con mejoras en el bienestar.

\section{Sincronía Emocional Percibida}

En línea con lo anterior, se ha argumentado que las emociones colectivas que se generan en rituales colectivos refuerzan los sentimientos de pertenencia, identificación con el grupo, cohesión y solidaridad (Durkheim, 1912/1995) y que la efervescencia colectiva -empíricamente medida como sincronía emocional percibida; ver Páez y cols. (2015)-, además de unir a los individuos con la comunidad, provee un marco para la creación de símbolos compartidos por la comunidad (Collins, 2004; Ismer, 2011).

Un claro ejemplo de la evocación de emociones positivas y efervescencia colectiva en los rituales colectivos es en las organizaciones 
religiosas, en las que la implementación de estos se mueve por el intercambio y creación de energía emocional (Collins, 2004) que fomenta la participación de los miembros de la congregación e incrementa la solidaridad entre sus miembros (Draper, 2014; Wollschleger, 2017) y se ha encontrado que brindan una sensación de elevación entre los miembros, así como una serie de reacciones fisiológicas, tales como piel de gallina o temblores (Inbody, 2015). Wellman, Corcoran y StocklyMeyerdirk (2014) refirieron que el establecimiento de rituales en organizaciones religiosas promovía altos niveles de energía, que podía ser expresado incluso como estar bajo el efecto de alguna droga; elevaba los sentimientos de pertenencia y aceptación entre sus miembros; cargaba los símbolos de pertenencia al grupo con significancia emocional; daban un sentido de propósito al grupo a través de los sentimientos de moralidad y una sensación de espiritualidad elevada.

Además de rituales religiosos existen otras fuentes de eventos colectivos que evocan emociones colectivas que han sido abordadas por la literatura, como los rituales de nacionalización para migrantes (Damsholt, 2018); rituales extremos como caminar sobre fuego (Fischer, et al., 2014) o ceremonias nacionalistas (Cui, 2018). En grandes eventos deportivos, como la Copa Mundial de Fútbol, la efervescencia colectiva ha sido asociada positivamente con la identificación nacional y la percepción positiva de los símbolos nacionales (Ismer, 2011; von Scheve et al., 2014). Los movimientos sociales también han sido objeto de análisis por la evocación de energía emocional en sus participantes, así como la práctica de rituales en marchas y otras expresiones. En una marcha silenciosa colectiva después de un ataque terrorista en Noruega se destacó que la participación en rituales colectivos fortalecía la identidad grupal y tenían una función cohesiva con el grupo, además de generar sentimientos de orgullo y calidez en la comunidad ( $\mathrm{H} \phi$ eg, 2015).

\section{Objetivo e Hipótesis}

El presente estudio está enmarcado en el análisis de los efectos psicosociales de awe a diversos niveles sociales, como el individual (e.g., 
bienestar) como colectivo (e.g., ayuda colectiva a grupos minoritarios). De manera específica, proponemos que la experiencia emocional de awe tendrá un efecto positivo sobre la identificación global (e.g., Bai et al., 2017), la conducta prosocial (e.g., Prade \& Saroglou, 2016), y el bienestar psicológico (e.g., Rudd, Vohs, \& Aaker, 2012) (H1). Además, tras analizar diferentes como dichas variables se pueden relacionar en un modelo más complejo, se espera un efecto secuencial desde la identificación con la humanidad a la conducta prosocial (Buchan, Jeong, \& Ward, 2017) y, a su vez, se prevé que proporcionar ayuda a otros se relacione con el bienestar de las personas (Curry et al., 2018).

Debido a que esta hipótesis únicamente hace referencia al rol de la emoción y no a las disposiciones personales, en este estudio, también se incluyen los valores de universalismo y de benevolencia. Basándonos en investigaciones previas, estos valores de trascendencia se vincularán con la identificación global (McFarland et al., 2019) y la conducta prosocial (Daniel, Bilgin, Brezina, Strohmeier, \& Vainre, 2015).

Por otra parte, también proponemos que la experiencia emocional evocada de awe puede estar relacionada y predecir un proceso emocional presente en una gran variedad de eventos colectivos, a saber, la percepción de sincronía emocional (Páez et al., 2015). Por esta razón, esperamos que sea la experiencia pasada y recordada de awe, y no las variables disposicionales de la personalidad apertura a la experiencia y extraversión, la que tenga un efecto sobre la percepción de Sincronía Emocional de los participantes en otros eventos colectivos pasados (H2).

\section{Método}

\section{Participantes}

La muestra total empleada para esta investigación consta de 652 participantes provenientes del País Vasco $(N=131)$ y de México $(N=$ 521 ), con un $65.2 \%$ de mujeres, y edades comprendidas entre 18 y 79 
Eventos locales, efectos globales: emociones trascendentes e identificación / Pizarro et al.

años $(M=25.77, D E=10.09)$. Para más detalles sobre la muestra, véase Tabla $1^{7}$.

\section{Tabla 1}

Caracteristicas sociodemográficas de las muestras del País Vasco y de México.

\begin{tabular}{lccc}
\hline & $\begin{array}{c}\text { País Vasco } \\
(n=131)\end{array}$ & $\begin{array}{c}\text { México } \\
(n=521)\end{array}$ & $\begin{array}{c}\text { Total } \\
(N=652)\end{array}$ \\
\hline Género & $64.6 \%$ & $65.4 \%$ & $65.2 \%$ \\
Femenino & & & \\
Nivel educativo & $1.6 \%$ & $3.1 \%$ & $2.8 \%$ \\
Primario completo & $14.4 \%$ & $8.3 \%$ & $9.5 \%$ \\
Secundario completo & $53.6 \%$ & $67.0 \%$ & $64.4 \%$ \\
Terciario completo & $30.4 \%$ & $20.6 \%$ & $23.1 \%$ \\
Universitario & & & \\
Estado civil & $62.4 \%$ & $70.2 \%$ & $68.7 \%$ \\
Soltero/a & $33.6 \%$ & $25 \%$ & $26.6 \%$ \\
Casado/a o convive en & $4 \%$ & $3.5 \%$ & $3.6 \%$ \\
Pareja & $0 \%$ & $1.3 \%$ & $1.1 \%$ \\
Separado/a o Divorciado & & & \\
Viudo/a & & & \\
Orientación política & & & \\
\hline
\end{tabular}

$7 \mathrm{Al}$ analizar las muestras, se estudió la posibilidad de la existencia de diferencias entre las muestras en función de las principales variables de estudio (género que fue contrastado en el análisis descriptivo de las muestras: $X_{(1)}^{2}=0.26, p=.872$ ). En primer lugar, y debido a que el foco principal de estudio es la intensidad de awe y su efecto en diversas variables (Valores de Trascendencia, Identidad global y Bienestar), meta-analizamos la relación de esta emoción con dichas variables criterio utilizando un modelo aleatorio (a través del paquete metafor para R; Viechtbauer, 2015). Todos los resultados indican que no existe heterogeneidad; es decir, no existe una fuente de error que suponga que los efectos de las asociaciones sean diferentes entre los participantes de País Vasco y México. En detalle, así lo señalan los resultados obtenidos en la relación de Awe con los Valores de Trascendencia $\left(Q_{(1)}=1.290, p=.256\right)$, con la Identidad global $\left(Q_{(1)}=1.350, p=.245\right)$, y con el Bienestar $\left(Q_{(1)}=0.096, p=.757\right)$. En base a la homogeneidad en la relación entre las variables criterio para ambas muestras, en adelante, todos los análisis se realizan de manera conjunta. 


\begin{tabular}{lccc}
\hline & $\begin{array}{c}\text { País Vasco } \\
(n=131)\end{array}$ & $\begin{array}{c}\text { México } \\
(n=521)\end{array}$ & $\begin{array}{c}\text { Total } \\
(N=652)\end{array}$ \\
\hline Izquierda & $73.6 \%$ & $22.6 \%$ & $32.7 \%$ \\
Centro & $20.8 \%$ & $48.8 \%$ & $43.3 \%$ \\
Derecha & $5.6 \%$ & $28.5 \%$ & $24 \%$ \\
Religión & & & \\
Católica & $42 \%$ & $84.6 \%$ & $76.9 \%$ \\
Evangélica o protestante & $0.9 \%$ & $2.1 \%$ & $1.9 \%$ \\
$\begin{array}{l}\text { Ateísmo, agnosticismo o } \\
\text { sin religión }\end{array}$ & $54.5 \%$ & $9.8 \%$ & $17.8 \%$ \\
Otra & $2.7 \%$ & $3.5 \%$ & $3.4 \%$ \\
\hline
\end{tabular}

\section{Medición}

\section{Fase I}

En esta fase, se incluyen tanto la evocación del estímulo de autotrascendencia (i.e., lista de eventos y la escala de awe, como también el resto de escalas criterio). El reporte de medias y de indicadores de fiabilidad se encuentran en la Tabla 2 y en la Tabla Suplementaria 1.

Lista de eventos emocionales (Cusi et al., 2018; Pizarro et al., 2018). Para evaluar los eventos que desencadenan las tres emociones de trascendencia, se empleó la lista de eventos pasados que agrupa elicitadores típicos de elevación (Cusi et al., 2018), de kama muta basada en la literatura (Fiske et al., 2017) y de awe (Pizarro et al., 2018). Los participantes leyeron los 44 eventos emocionales y marcaron una " $X$ " en caso de haber vivido dicho evento durante los últimos tres meses, y dejaron en blanco el resto de las casillas cuyas situaciones no se presenciaron.

Awe (Asombro Maravillado, de Pizarro et al., 2018). Para evaluar la experiencia de asombro maravillado se utilizó la Escala de Asombro Maravillado (Pizarro et al., 2018) que evalúa mediante 16 ítems en formato Likert de 1 (Nada) a 7 (Mucho), la experiencia característica 
y habitual, así como la intensidad, de asombro maravillado, a través de cinco dimensiones; estímulos y evaluaciones (e.g., Me siento en presencia de algo grandioso), tendencia de acción (e.g., Deseo formar parte de algo más grande que uno), y respuestas afectivas (e.g., Me siento intensamente emocionado ante ello), fisiológicas (e.g., Tengo una sensación de sobrecogimiento ante algo grandioso), y cognitivas (e.g., Siento que formo parte de algo más grande, mayor que uno mismo).

Valores (Schwartz, 1994). Se aplicaron 5 items de la dimensión de auto-trascendencia, compuesta por tres ítems para los valores universalistas que hacen referencia a la promoción de la justicia, la tolerancia y la protección para el bienestar de todas las personas y de la naturaleza (e.g., Es importante para éllella ser tolerante con todo tipo de personas y grupos), y dos ítems para los valores de benevolencia comprendidos como la preservación y mejora del bienestar del endogrupo, favoreciendo el apoyo social y la cooperación (e.g., Es muy importante para éll ella ayudar a la gente que lella rodea. Se preocupa por su bienestar). Cada persona debía indicar qué tanto creía parecerse a la persona descrita, en una escala de 1 (No se parece en nada a mí) a 6 (Se parece mucho a mí).

Cinco Grandes Rasgos de Personalidad (basada en la escala Big Five Inventory de John, Donahue, \& Kentle, 1991; versión en español de Benet-Martínez \& John, 1998). Se emplearon las dimensiones de apertura (8 ítems; e.g., Tiene intereses muy diversos) y extraversión (5 ítems; e.g., Es extrovertidola, sociable), en una escala de 1 (Muy en desacuerdo) a 5 (Muy de acuerdo).

Identidad Global (basada en la escala Global Identity de Der-Karabetian \& Ruiz, 1997). Se aplicaron 4 ítems, en un rango de respuesta de 1 (Muy en desacuerdo) a 6 (Muy de acuerdo), mide la identificación supranacional con toda la humanidad como una comunidad global (e.g., Pienso que soy ciudadano del mundo).

Conducta prosocial en el pasado (ad-hoc). Esta escala indaga mediante 3 ítems la participación en el pasado en manifestaciones, ONG's e instituciones que colaboran con temas humanitarios y ambientales (e.g., En el pasado ha participado en ONG's de carácter humanitario), en una escala de 0 (Nunca) a 4 (Con mucha frecuencia). 
Bienestar (Pemberton Happiness Index de Hervás \& Vázquez, 2013). A través de 6 ítems, se evalúan la dimensión de bienestar eudaimónico en una escala de 10 puntos $(0=$ Totalmente en desacuerdo a 10 = Totalmente de acuerdo), (e.g., Me siento capaz de resolver la mayoría de los problemas de mi día a día).

\section{Fase II}

Esta fase comenzó con una segunda evocación del recuerdo, la cual se centraba a actividades colectivas recientes que podían evocar Sincronía Emocional Percibida.

Descripción de una actividad colectiva importante (ad-hoc). Se pidió a los participantes que escribieran en cinco líneas una de las actividades colectivas o grupales más importantes en las que usualmente participan (e.g., Reuniones familiares, grupo politico, misa semanal, manifestaciones...). Era imprescindible que los participantes contestaran a este ítem, dado que la descripción de dicha actividad compromete las respuestas proporcionadas en la escala subsiguiente.

Sincronía Emocional Percibida - SEP de aquí en adelante (versión corta de Perceived Emotional Synchrony Páez, Rimé, Basabe, Wlodarczyk, \& Zumeta, 2015). Se compone de 6 ítems que miden la percepción de haber vivido una emoción intensa de manera colectiva (e.g., Hemos sentido una fuerte emoción compartida), en un formato de 7 puntos ( 1 = Nada, 7 = Mucho).

\section{Procedimiento}

El estudio se realizó con una metodología cuantitativa y un diseño correlacional de tipo encuesta. Los datos fueron obtenidos mediante la aplicación de un cuestionario retrospectivo de auto-informe, basado en un recuerdo emocional libre, que se llevó a cabo durante el segundo semestre de 2019. En él, los participantes tenían que indicar en una lista cerrada (ver Anexos) de 44 eventos que pueden suscitar las emociones de conmovido por amor al prójimo, asombro maravillado y elevación moral (basados en Pizarro et al. 2018, y Cusi et al., 2018), y se les pidió 
que indicarán si en los últimos tres meses habían vivido o atestiguado alguno de estos sucesos emocionales. A continuación, cada participante debía relatar uno de ellos en mayor profundidad y posteriormente, contestar la escala de awe y el resto del cuestionario que incluía las variables criterio (i.e., de identidad, valores, etc.) ${ }^{8}$.

La participación en este estudio fue voluntaria, sin remuneración, $\mathrm{y}$, en promedio tardaron unos 40-45 minutos. Asimismo, todos los procedimientos empleados en este estudio garantizan la confidencialidad de las respuestas y están conforme con los requisitos éticos del comité de ética de investigación en humanos y de la declaración de Helsinki de 1964.

\section{Análisis estadísticos}

Luego de analizar posibles fuentes de heterogeneidad (ver nota 1), se exploraron las estructuras factoriales de las escalas empleadas. Para esto, se realizaron diversos análisis factoriales confirmatorios, a la vez que se reportan los índices de confiabilidad (Omega de McDowell). Luego, se realizaron análisis de correlaciones de Pearson entre las variables de interés, y finalmente, modelos de ecuaciones estructurales. Es últimos, al igual que la evaluación de las estructuras factoriales, con el paquete lavaan (Rosseel, 2014) en R (R Core Team, 2014) con RStudio (RStudio Team, 2015).

\section{Resultados}

\section{Análisis de Correlaciones}

La Tabla 1 muestra los análisis de correlaciones entre las principales variables. Los resultados indican que la emoción trascendente de

8 En este estudio solo se reportan los datos relacionados con awe, sin embargo, se aplicaron también otras escalas las cuales no se consideran haber afectado los resultados reportados en este artículo. 
awe se asocia de forma positiva con todas las variables de estudio salvo la edad, con un intervalo de correlaciones que oscila entre $r=.09$ para el sexo, a $r=.35$ para los valores de universalismo. Entre los valores de asociación más altos (i.e., mayores que .30) se encuentran los valores de benevolencia y la Sincronía Emocional Percibida (SEP).

De forma similar, la Identidad global se asocia a todas las variables de estudio salvo con la edad, con un intervalo de correlaciones que oscila entre $r=.14$ con conducta prosocial, a $r=.40$ con bienestar y para el caso de la asociación con SEP, una asociación media-alta de $r=$ .35. La conducta prosocial, por otra parte, tiene un rango más amplio de asociaciones estadísticamente, desde $r=-.10$ con la edad, hasta $r=.30$ con SEP, siendo sexo la única variable con la que no muestra relación. Al mismo tiempo, bienestar, se asocia de forma positiva y significativa con todas las variables salvo con sexo, en un intervalo que oscila desde $r=.11$ con conducta prosocial, hasta $r=.40$ con valores benevolentes. Respecto a SEP, el intervalo de asociaciones fue de $r=.17$ para extraversión hasta $r=.35$ para benevolencia.

\section{Modelos de ecuaciones estructurales (SEM)}

En el primer modelo representado en la Figura 1, se puede observar los efectos directos, significativamente positivos y de intensidad mediaalta de awe sobre la identidad global y el bienestar. Se observa que la emoción tiene un efecto positivo, significativo y directo en la identidad global y en el bienestar, y no tiene un efecto directo en la conducta prosocial pasada. Por otra parte, se observa que la identificación con la humanidad también tiene un impacto positivo en la ayuda pasada y que esta, tiene un efecto positivo (i.e., marginalmente significativo) en el bienestar auto-reportado. Los indicadores de bondad de ajuste del modelo global son satisfactorios $\left(X_{(450)}^{2}=736.78 ; p<0.001\right.$; CFI = $.953 \mathrm{TLI}=.948, \mathrm{RMSEA}=.04290 \% \mathrm{CI}[.037, .047])$. 


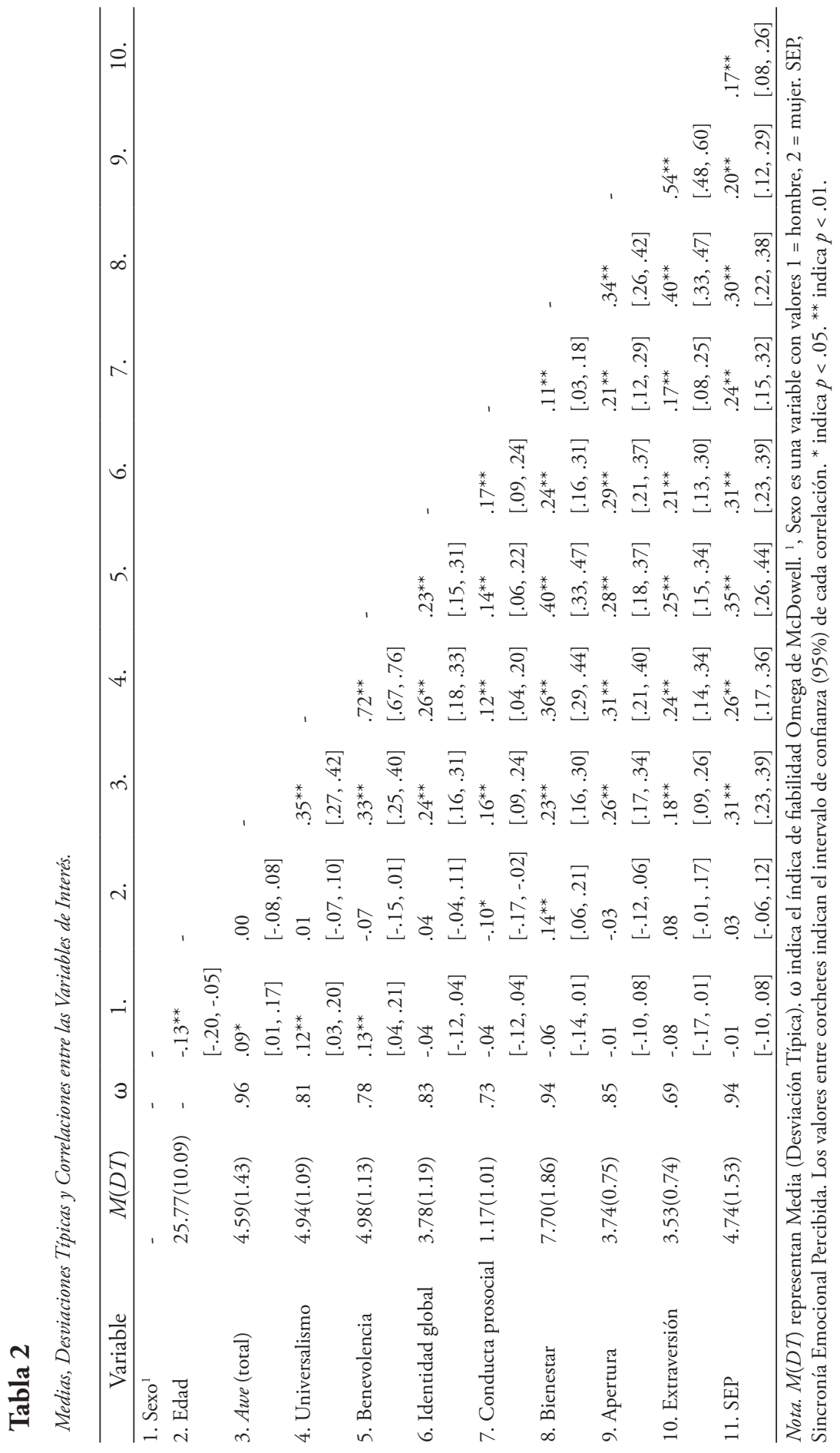




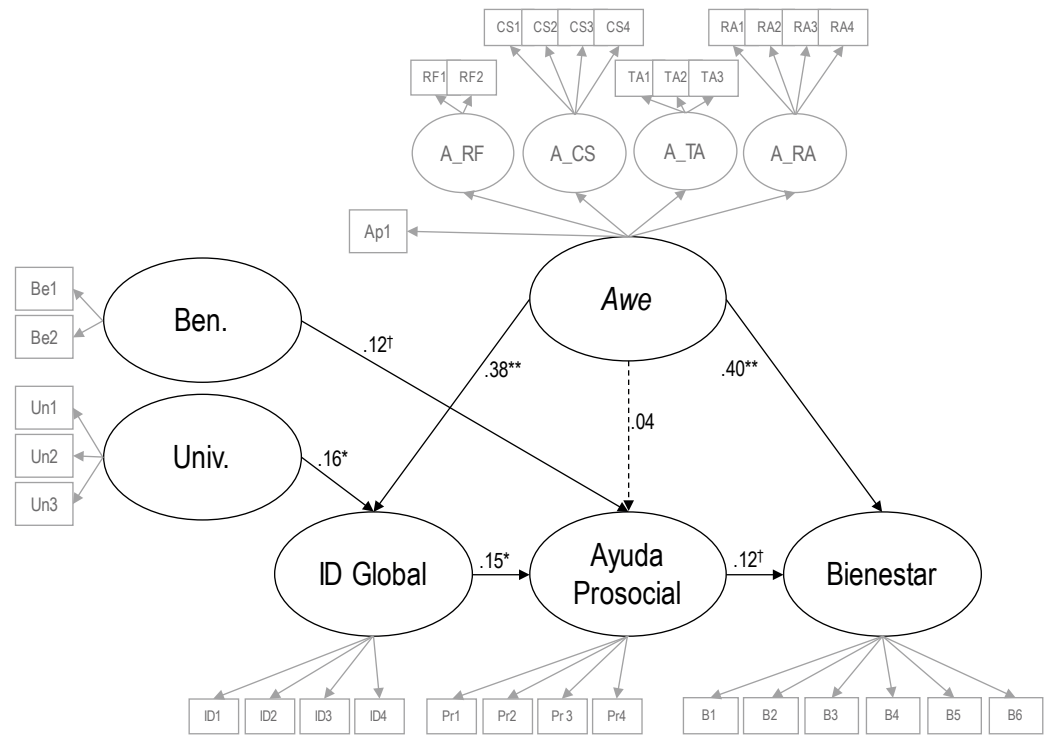

Figura 1. Efectos de Awe en Identidad Global, Ayuda Prosocial y Bienestar. Nota: ${ }^{\dagger}, p<.10 ;{ }^{*}, p<.01 ;{ }^{* *}, p<.001$.

En la Figura 2, se muestra el modelo que busca explicar la experiencia de la SEP. En este sentido, las características de personalidad más vinculadas a las relaciones interpersonales y eventos colectivos, como son la apertura a la experiencia y la extraversión, no tienen efectos directos significativos sobre SEP. Sin embargo, sí muestran un efecto positivo y significativo los valores de universalismo y, con mayor fuerza, awe. Los indicadores de bondad de ajuste del modelo global son muy adecuados $\left(X_{(450)}^{2}=932.82 ; p<0.001 ; \mathrm{CFI}=.944 \mathrm{TLI}=.939\right.$, RMSEA $=.04190 \%$ CI $[.040, .050])$. 


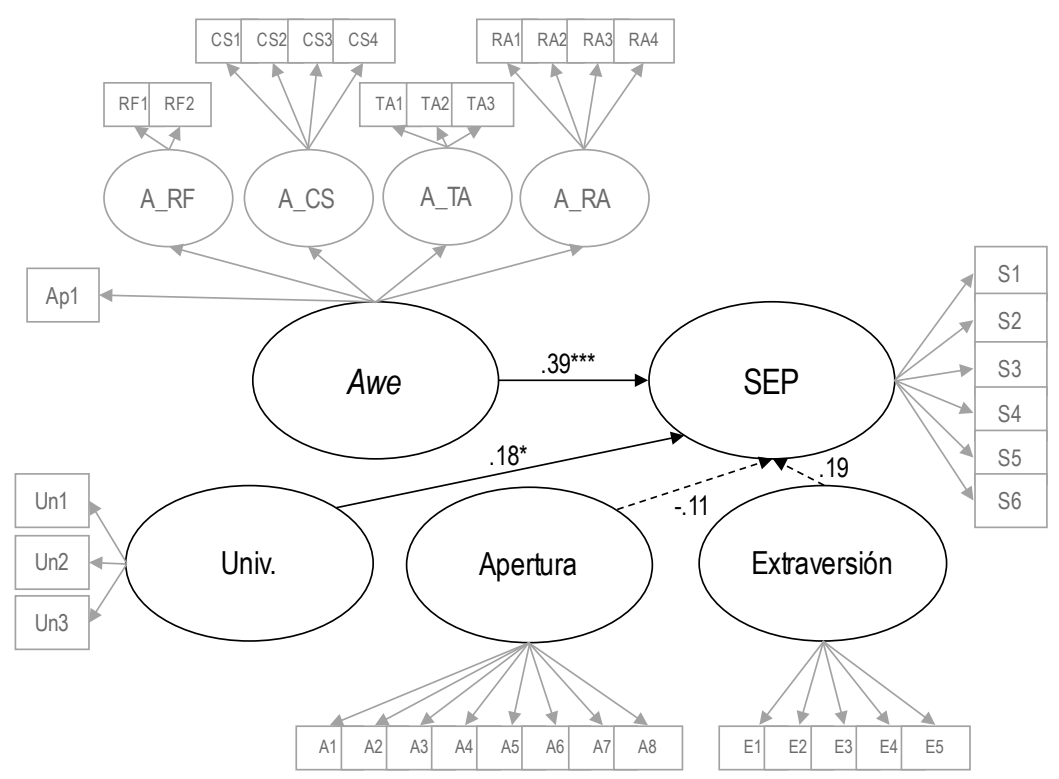

Figura 2. Sincronía Emocional Percibida explicada en función de Awe, Universalismo y Apertura. Nota: $p<.05$; $^{* *}, p<.01$; $^{* *}, p<.001$

\section{Discusión}

Los resultados de este proyecto de investigación sugieren que el alcance de nuestras experiencias emocionales es, en general, más fuerte y social de lo que se podría pensar. En detalle, la experiencia de una emoción de auto-trascendencia sugiere que tanto sus efectos positivos desde una perspectiva funcional van más allá del individuo y diada, sino que impactan el nivel colectivo y supra-categorial.

En relación con $\mathrm{H} 1$, se observó que la experiencia emocional de awe tiene un impacto positivo en la identificación global y el bienestar eudaimónico reportado por participantes, lo que va en línea por lo propuesto por otros autores (Bai et al., 2017; Rudd et al., 2012). Sin embargo, 
aunque existe una correlación entre awe y la conducta prosocial no logró probarse un efecto directo de la emoción en la prosocialidad, sino a través de la identificación con la humanidad. Además, la identificación global, la conducta prosocial y el bienestar eudaimónico tienen efectos secuenciales entre ellas que en última instancia implica una mejora en el bienestar de las personas. En otras palabras, vivir momentos de autotrascendencia implica una mayor apertura más hacia otros y, por ende, queramos formar parte en grupos más inclusivos, ayudar a otras personas y finalmente percibamos un grado mayor de desarrollo y bienestar.

En detalle, las correlaciones y análisis de ecuaciones estructurales mostraron que es el aspecto emocional -en oposición a una perspectiva que explique la identificación o la ayuda prosocial en base a rasgos de personalidad o diferencias individuales- lo que motiva la identificación con todas las personas. Además, a través de esta identificación, es posible ayudar a otras personas (Buchan et al., 2017) y también nos reporta una mejora en el bienestar (Curry et al., 2018). Así, se puede confirmar el carácter transcendental de awe (ver Pizarro et al., 2018) a la vez que se analiza su rol en una serie de variables de gran relevancia para la vida individual y social. Si bien el aspecto específico del efecto de awe en la ayuda prosocial pasada no es significativo, cabe destacar la vía indirecta i.e., (a través de la identificación global) y que, en un análisis posterior, awe sí explica la conducta prosocial cuando no existen otras variables, por lo que se cumple H1. En general, se destaca un aspecto emocional en los procesos de formación de identidades, de ayuda y también, del retorno que implica para las personas (Bai et al., 2017; Stellar et al., 2017; Van Cappellen \& Rimé, 2014), y, de manera importante, se comprueba también la relación secuencial entre estas.

Estos resultados no pretenden cuestionar, por otra parte, el poder explicativo de ciertas tendencias individuales estables en el tiempo. De hecho, en investigaciones previas se observa que los valores de universalismo y benevolencia se asociaron positivamente con la identificación global y la conducta prosocial (Daniel, Bilgin, Brezina, Strohmeier, \& Vainre, 2015; McFarland et al., 2019) y mostraron un efecto directo también en el modelo de ecuaciones estructurales en este estudio. Todo 
esto sugiere que la disposición a la tolerancia, la lealtad, la justicia y el cuidado de la naturaleza favorece sentirse parte del mundo y ayudar a otros, y, además, la vivencia emocional continúa aportando un poder explicativo único.

Con respecto a $\mathrm{H} 2$, es posible ver que awe puede también explicar un proceso de base emocional, como lo es la SEP. En detalle, una experiencia emocional de auto-trascendencia predice los puntajes de haber compartido la misma emoción e intensidad con otras personas, inclusive en un evento intrínsecamente diferente, pero también con base social. Además, y al igual que el primer modelo, el poder explicativo de awe es superior a lo explicado por factores más estables de personalidad (e.g., valores y rasgos).

En general, podemos concluir que la experiencia de awe es una experiencia emocional de auto-trascendencia del yo que a) motiva el fortalecimiento de identidades más inclusivas, b) aumenta (indirectamente) las formas colectivas de ayuda a otros, c) implica una relación positiva con el bienestar eudaimónico de las personas, y d) es capaz de predecir un proceso emocional, con efectos emergentes (i.e., SEP; ver Páez et al., 2015).

El presente estudio cuenta con algunas limitaciones que es necesario hacer notar. En primer lugar, la toma de datos se realizó de forma transversal, lo que implica la necesidad de realizar estudios longitudinales y experimentales para probar las hipótesis con una mayor validez predictiva. Otro aspecto que considerar es que la muestra en su mayoría se conformó por jóvenes, lo que pudo hacer que la edad no resultara una variable significativa en muchas de las relaciones estadísticas; por lo tanto, será necesario incorporar muestras con población adulta y adulta mayor para identificar con mayor certeza si la edad pudiera ser un factor que modere o medie entre las relaciones de las emociones con la identificación global y el bienestar eudaimónico.

Cabe destacar que no se encontraron diferencias significativas entre diversas naciones (i.e., País Vasco y México) en las variables incluidas en este estudio, por lo que podemos suponer que la relación entre los procesos emocionales descritos, la construcción de la identidad global y 
el bienestar eudaimónico opera de forma similar en sociedades desarrolladas y en proceso de desarrollo, lo que podría dar luz para la creación de rituales que fomenten emociones de trascendencia celebrando temas globales y que pudieran impactar en diferentes escenarios. De cara al futuro, podemos mencionar que awe y posiblemente, otras EAs son una vía de gran interés para el estudio de procesos colectivos que a su vez puedan ayudarnos a tratar temas que implican la colaboración y trabajo conjunto de diversas naciones y pueblos. Esta ayuda, a su vez, puede ser canalizada a través de la celebración de rituales y eventos colectivos que, a través de la benevolencia y universalismo ayuden a crear experiencias emocionales positivas que a su vez generan una cascada de fenómenos psicosociales positivos (de Rivera, 2018; de Rivera \& Carson, 2015).

\section{Referencias}

Algoe, S. B., \& Haidt, J. (2009). Witnessing excellence in action: the 'other-praising' emotions of elevation, gratitude, and admiration. J Posit Psychol., 4(2), 105-127. https://doi. org/10.1080/17439760802650519

Arnett, J. J. (2002). The Psychology of Globalization. American Psychologist, 57(10), 774-783. https://doi.org/10.1037//0003066X.57.10.774

Bai, Y., Maruskin, L. A., Chen, S., Gordon, A. M., Stellar, J. E., McNeil, G. D., ... Keltner, D. (2017). Awe, the Diminished Self, and Collective Engagement: Universals and Cultural Variations in the Small Self. Journal of Personality and Social Psychology, 113(2), 185-209. https://doi.org/10.1037/pspa0000087

Benet-Martínez, V., \& Hong, Y. (2014). The Oxforfd Handbook of Multicultural Identity. (V. Benet-Martínez \& Y. Hong, Eds.). New York: Oxford University Press.

Buchan, N. R., Brewer, M. B., Grimalda, G., Wilson, R. K., Fatas, E., \& Foddy, M. (2011). Global social identity and global cooperation. Psychological Science, 22(6), 821-828. https://doi. org/10.1007/s10922-011-9211-3 
Buchan, N. R., Jeong, S. S., \& Ward, A. K. (2017). Local Reasons to Give Globally: Identity Extension and Global Cooperation. Scientific Reports, 7(1), 1-8. https://doi.org/10.1038/ s41598-017-15683-0

Carlo, G., Okun, M. A., Knight, G. P., \& de Guzman, M. R. T. (2005). The interplay of traits and motives on volunteering: Agreeableness, extraversion and prosocial value motivation. Personality and Individual Differences, 38(6), 1293-1305. https://doi.org/10.1016/j.paid.2004.08.012

Cova, F., Deonna, J. A., \& Sander, D. (2015). Is elevation a way of being moved? Unpublished manuscript.

Cox, K. S. (2010). Elevation predicts domain-specific volunteerism 3 months later. The Journal of Positive Psychology, 5(5), 333-341. https://doi.org/10.1080/17439760.2010.507468

Curry, O. S., Rowland, L. A., Van Lissa, C. J., Zlotowitz, S., McAlaney, J., \& Whitehouse, H. (2018). Happy to help? A systematic review and meta-analysis of the effects of performing acts of kindness on the well-being of the actor. Journal of Experimental Social Psychology, 76(February), 320-329. https://doi.org/10.1016/j. jesp.2018.02.014

Cusi, O., Pizarro, J. J., Alfaro-Beracoechea, L., Vera-Calzaretta, A., González-Burboa, A., Carrera, P., \& Páez, D. (2018). Elevación o Inspiración Moral: Creación y Validación de una Escala de Elevación en Castellano [Moral Elevation or Inspiration: Creation and Validation of an Elevation Scale in Spanish]. Revista Latinoamericana de Psicología Positiva, 4, 38-56. Retrieved from http://psycap.cl/wp-content/uploads/2018/05/MonográficoRLPP-Mayo-2018_Versión-Final.pdf

Daniel, E., Bilgin, A. S., Brezina, I., Strohmeier, C. E., \& Vainre, M. (2015). Values and helping behavior: A study in four cultures. International Journal of Psychology, 50(3), 186-192. https://doi. org/10.1002/ijop.12086 
de Rivera, J. (2018). Themes for the celebration of global community. Peace and Conflict: Journal of Peace Psychology, 24(2), 216-244. https://doi.org/http://dx.doi.org/10.1037/pac0000340

de Rivera, J., \& Carson, H. A. (2015). Cultivating a Global Identity. Journal of Social and Political Psychology, 3(2), 310-330. https:// doi.org/10.5964/jspp.v3i2.507

Der-Karabetian, A., \& Ruiz, Y. (1997). Affective Bicultural and GlobalHuman Identity Scales for Mexican-American Adolescents. Psychological Reports, 80, 1027-1039. https://doi.org/10.2466/ pr0.1997.80.3.1027

Fischer, A. H., \& Manstead, A. S. R. (2008). Social functions of emotion. In M. Lewis, J. Haviland-Jones, \& L. Feldman Barrett (Eds.), Handbook of emotions (pp. 456-468). New York, London: The Guilford Press. https://doi.org/10.2307/2076468

Fiske, A. P. (1992). The four elementary forms of sociality: Framework for a unified theory of social relations. Psychological Review, 99(4), 689-723. https://doi.org/10.1037/0033-295X.99.4.689

Fiske, A. P., Schubert, T. W., \& Seibt, B. (2016). "Kama Muta” or 'Being Moved by Love': A Bootstrapping Approach to the Ontology and Epistemology of an Emotion. In J. Cassanity \& U. Menon (Eds.), Universalism without uniformity: Explorations in mind and culture. Chicago: University of Chicago Press. Retrieved from http://kamamutalab.org/wp-content/uploads/2016/10/ Epistemology_ontology_of_kama_muta_chapter_in_press.pdf

Fiske, A. P., Seibt, B., \& Schubert, T. W. (2017). The Sudden Devotion Emotion: Kama Muta and the Cultural Practices Whose Function Is to Evoke It. Emotion Review, 1-13. https://doi. org/10.1177/1754073917723167

Frankl, V. E. (1966). Self-Transcendence as a Human Phenomenon. Journal of Humanistic Psychology, 6(2), 97-106. https://doi. org/10.1177/002216786600600201

Fredrickson, B. L. (2013). Positive Emotions Broaden and Build. In P. Devine \& A. Plant (Eds.), Advances in Experimental Social 
Psychology (1st ed., Vol. 47, pp. 1-53). Burlington: Academic Press. https://doi.org/10.1016/B978-0-12-407236-7.00001-2 Greenaway, K. H., \& Louis, W. R. (2010). Only human: Hostile human norms can reduce legitimization of intergroup discrimination by perpetrators of historical atrocities. British Journal of Social Psychology, 49(4), 765-783. https://doi. org/10.1348/014466609X479202

Greenaway, K. H., Quinn, E. A., \& Louis, W. R. (2011). Appealing to common humanity increases forgiveness but reduces collective action among victims of historical atrocities. European Journal of Social Psychology, 41(5), 569-573. https://doi.org/10.1002/ ejsp.802

Guan, F., Chen, J., Chen, O., Liu, L., \& Zha, Y. (2019). Awe and prosocial tendency. Current Psychology. https://doi.org/10.1007/ s12144-019-00244-7

Habashi, M. M., Graziano, W. G., \& Hoover, A. E. (2016). Searching for the Prosocial Personality: A Big Five Approach to Linking Personality and Prosocial Behavior. Personality and Social Psychology Bulletin, 42(9), 1177-1192. https://doi. org/10.1177/0146167216652859

Haidt, J. (2000). The Positive Emotion of Elevation. Prevention \& Treatment, 3(3), 1-5. https://doi.org/10.1080/10481885.2010 .514828

Haidt, J. (2003a). Elevation and the positive psychology of morality. In C. L. M. Keyes \& J. Haidt (Eds.), Flourishing: Positive psychology and the life well lived (pp. 275-289). Charlottesville, VA: American Psychological Association.

Haidt, J. (2003b). The Moral Emotions. In R. J. Davidson, K. R. Scherer, \& H. H. Goldsmith (Eds.), Handbook of affective sciences (pp. 852-870). Oxford: Oxford University Press. https://doi. org/10.1093/mnras/stx 1358

Hervás, G., \& Vázquez, C. (2013). Construction and validation of a measure of integrative well-being in seven languages: The Pem- 
berton Happiness Index. Health and Quality of Life Outcomes, 11(1), 1-13. https://doi.org/10.1186/1477-7525-11-66

Hornsey, M. J. (2008). Social Identity Theory and Self-categorization Theory: A Historical Review. Social and Personality Psychology Compass, 2(1), 204-222. https://doi. org/10.1111/j.1751-9004.2007.00066.x

Hornsey, M. J., \& Hogg, M. A. (1999). Subgroup differentiation as a response to an overly-inclusive group: A test of optimal distinctiveness theory. European Journal of Social Psychology, 29(4), 543-550. https://doi.org/10.1002/(SICI) 1099-0992(199906)2 9:4<543::AID-EJSP945>3.0.CO;2-A

Hornsey, M. J., \& Hogg, M. A. (2002). The effects of status on subgroup relations. British Journal of Social Psychology, 41, 203-218. https://doi.org/doi.org/10.1348/014466602760060200

Keltner, D., \& Haidt, J. (1999). Social functions of emotions at four levels of analysis. Cognition and Emotion, 13(5), 505-521. https://doi.org/10.1080/026999399379168

Keltner, D., \& Haidt, J. (2003). Approaching awe a moral spiritual and aesthetic emotion. Cognition and Emotion, 17(2), 297-314. https://doi.org/10.1080/02699930244000318

Liu, J. H., \& Macdonald, M. (2016). Towards a Psychology of Global Consciousness Through an Ethical Conception of Self in Society. Journal for the Theory of Social Behaviour, 46(3), 310334. https://doi.org/10.1111/jtsb.12101

Markus, H. R., \& Kitayama, S. (1991). Culture and the Self: Implications for Cognition, Emotion, and Motivation. Psychological Review, 98(2), 224-253. https://doi.org/10.1037/0033-295X.98.2.224

Markus, H. R., \& Kitayama, S. (2010). Cultures and selves: A cycle of mutual constitution. Perspectives on Psychological Science, 5(4), 420-430. https://doi.org/10.1177/1745691610375557

Maslow, A. H. (1964). Religions, Values, and Peak Experiences. New York: Penguin.

McFarland, S., Hackett, J., Hamer, K., Katzarska-Miller, I., Malsch, A., Reese, G., \& Reysen, S. (2019). Global Human Identification and Citizenship: A Review of Psychological Studies. Advances in 
Political Psychology, 4O(S1), 141-171. https://doi.org/10.1111/ pops. 12572

McFarland, S., Webb, M., \& Brown, D. (2012). All humanity is my ingroup: A measure and studies of identification with all humanity. Journal of Personality and Social Psychology, 103(5), 830-853. https://doi.org/10.1037/a0028724

Nickerson, A. M., \& Louis, W. R. (2008). Nationality Versus Humanity? Personality, Identity, and Norms in Relation to Attitudes Toward Asylum Seekers. Journal of Applied Social Psychology, 38(3), 796817. https://doi.org/10.1111/j.1559-1816.2007.00327.x

Niedenthal, P. M., \& Brauer, M. (2012). Social Functionality of Human Emotion. Annual Review of Psychology, 63, 259-285. https://doi.org/10.1146/annurev.psych.121208.131605

Pierre, J. (2019). Putting the "Move" in Social Movements: Assessing the Role of Kama Muta in Online Activism. In Information in Contemporary Society. iConference 2019. Lecture Notes in Computer Science, vol 11420. (pp. 365-376). Cham: Springer. https://doi.org/10.1007/978-3-030-15742-5_35

Piff, P. K., Dietze, P., Feinberg, M., Stancato, D. M., \& Keltner, D. (2015). Awe , the Small Self, and Prosocial Behavior. Journal of Personality and Social Psychology, 108(6), 883-899.

Pizarro, J. J., Cusi, O., Alfaro-Beracoechea, L., González-Burboa, A., Vera-Calzaretta, A., Carrera, P., \& Páez, D. (2018). Asombro maravillado, temor admirativo o respeto sobrecogido: Revisión teórica y Validación de una Escala de Asombro en Castellano [Awe: Review of theory and Validation of an Awe Scale in Spanish]. Revista Latinoamericana de Psicología Positiva, 4, 57-76. Retrieved from http://psycap.cl/wp-content/uploads/2018/05/ Monográfico-RLPP-Mayo-2018_Versión-Final.pdf

Pohling, R., \& Diessner, R. (2016). Moral elevation and moral beauty: A review of the empirical literature. Review of General Psychology, 20(4), 412-425. https://doi.org/10.1037/gpr0000089 
R Core Team. (2014). R: A language and environment for statistical computing. Vienna, Austria: R Foundation for Statistical Computing.

Reysen, S., \& Katzarska-Miller, I. (2013). A model of global citizenship: Antecedents and outcomes. International Journal of Psychology, 48(5), 858-870. https://doi.org/10.1080/00207594.2012.701 749

Roccas, S., \& Brewer, M. B. (2002). Social Identity Complexity. Personality and Social Psychology Review, 6(2), 88-106.

Rosenmann, A., Reese, G., \& Cameron, J. E. (2016). Social Identities in a Globalized World: Challenges and Opportunities for Collective Action. Perspectives on Psychological Science, 11(2), 202-221. https://doi.org/10.1177/1745691615621272

Rosseel, Y. (2014). lavaan: an R package for structural equation modeling. Journal of Statistical Software, 48(2), 1-21. https:// doi.org/10.18637/jss.v048.i02

RStudioTeam. (2015). RStudio: Integrated Development for R. Boston, MA: RStudio, Inc. Retrieved from http://www.rstudio.com/

Schubert, T. W., Zickfeld, J. H., Seibt, B., \& Fiske, A. P. (2018). Moment-to-moment changes in feeling moved match changes in closeness, tears, goosebumps, and warmth: time series analyses. Cognition and Emotion, 32(1), 174-184. https://doi.org/10.108 0/02699931.2016.1268998

Schurtz, D. R., Blincoe, S., Smith, R. H., Powell, C. A. J., Combs, D. J. Y., \& Kim, S. H. (2012). Exploring the social aspects of goose bumps and their role in awe and envy. Motivation and Emotion, 36(2), 205-217. https://doi.org/10.1007/s11031-011-9243-8

Schwartz, S. H. (1994). Are There Universal Aspects in the Structure and Contents of Human Values? Journal of Social Issues, 50(4), 19-45. https://doi.org/10.1111/j.1540-4560.1994.tb01196.x

Seibt, B., Schubert, T. W., Zickfeld, J. H., \& Fiske, A. P. (2018). Touching the base: heart-warming ads from the 2016 U.S. election moved viewers to partisan tears. Cognition and Emotion, O(0), 1-16. https://doi.org/10.1080/02699931.2018.1441128 
Shiota, M. N., Keltner, D., \& Mossman, A. (2007). The nature of awe: Elicitors, appraisals, and effects on self-concept. Cognition \& Emotion, 21(5), 944-963. https://doi. org/10.1080/02699930600923668

Stellar, J. E., Gordon, A. M., Piff, P. K., Cordaro, D., Anderson, C. L., Bai, Y., ... Keltner, D. (2017). Self-Transcendent Emotions and Their Social Functions: Compassion, Gratitude, and Awe Bind Us to Others Through Prosociality. Emotion Review, 9(3), 200207. https://doi.org/10.1177/1754073916684557

Tajfel, H., \& Turner, J. C. (1979). An Integrative Theory of Intergroup Conflict. In W. G. Austin \& S. Worchel (Eds.), The social psychology of intergroup relations (pp. 33-47). Monterrey, CA: Brooks/Cole.

Thomson, A. L., \& Siegel, J. T. (2013). A moral act, elevation, and prosocial behavior: Moderators of morality. Journal of Positive Psychology, 8(1), 50-64. https://doi.org/10.1080/17439760.20 12.754926

Thomson, A. L., \& Siegel, J. T. (2017). Elevation: A review of scholarship on a moral and other-praising emotion. Journal of Positive Psychology, 12(6), 628-638. https://doi.org/10.1080/17 439760.2016.1269184

Turner, J. C., Hogg, M. A., Oakes, P. J., Reicher, S., \& Wetherell, M. S. (1987). Rediscovering the Social Group: A Self-Categorization Theory. Ney York: Blackwell. https://doi.org/10.2307/2073157 Urzúa, A., Basabe, N., Pizarro, J. J., \& Ferrer, R. (2017). Afrontamiento del estrés por aculturación: Inmigrantes latinos en Chile. Universitas Psychologica. https://doi.org/10.11144/Javeriana. upsy16-5.aeai

Van Cappellen, P., \& Rimé, B. (2014). Positive emotions and selftranscendence. In V. Saroglou (Ed.), Religion, personality, and social behavior (pp. 123-145). New York: Psychology Press. https://doi.org/10.4324/9780203125359

Wohl, M. J. A., \& Branscombe, N. R. (2005). Forgiveness and Collective Guilt Assignment to Historical Perpetrator Groups 
Depend on Level of Social Category Inclusiveness. Journal of Personality and Social Psychology, 88(2), 288-303. https://doi. org/10.1037/0022-3514.88.2.288

Yaden, D. B., Haidt, J., Hood, R. W., Vago, D. R., \& Newberg, A. B. (2017). The varieties of self-transcendent experience. Review of General Psychology, 21(2), 143-160. https://doi.org/10.1037/ gpr0000102

Zacarés, J. J., Iborra, A., Tomás, J. M., \& Serra, E. (2009). El desarrollo de la identidad en la adolescencia y adultez emergente: Una comparación de la identidad global frente a la identidad en dominios específicos. Anales de Psicología, 25(2), 316-329. https://doi.org/http://dx.doi.org/10.6018/analesps

Zickfeld, J. H., \& Schubert, T. W. (2018). Warm and touching tears: tearful individuals are perceived as warmer because we assume they feel moved and touched. Cognition and Emotion, 9931, 1-9. https://doi.org/10.1080/02699931.2018.1430556

Zickfeld, J. H., Schubert, T. W., Seibt, B., Blomster, J. K., Arriaga, P., Basabe, N., ... Fiske, A. P. (2018). Kama Muta: Conceptualizing and Measuring the Experience Often Labelled Being Moved Across 19 Nations and 15 Languages. Emotion, 19(3), 402-424. https://doi.org/10.1037/emo0000450

Zickfeld, J. H., Schubert, T. W., Seibt, B., \& Fiske, A. P. (2019). Moving Through the Literature: What Is the Emotion Often Denoted Being Moved? Emotion Review. https://doi. org/10.1177/1754073918820126

Recibido: 4 de marzo, 2020 Revisado: 28 de febrero, 2021 Aceptado: 3 de marzo, 2021 